\title{
Corticosteroid administration in oral and orthognathic surgery
}

\author{
Abstracted from \\ Dan AE, Thygesen TH, Pinholt EM. \\ Corticosteroid administration in oral and orthognathic surgery: \\ a systematic review of the literature and meta-analysis. J Oral Maxillofac Surg 2010; 68: 2207-2220. \\ Address for correspondence: Dr Dan, Department of Oral and Maxillofacial Surgery, \\ Institute of Odontology, Faculty of Health Science, University of Copenhagen, Blegdamsvej 3B, \\ 2200 Copenhagen N, Seeland, Denmark. E-mail: dan_anne@hotmail.com
}

\section{Question: In patients undergoing oral and orthognathic surgery does corticosteroid (CS) administration decrease oedema and pain and promote neuroregeneration?}

Data sources Medline and Cochrane Library.

Study selection Clinical trials of oral and orthognathic surgery involving CS administration.

Data extraction and synthesis The administered doses of CS in the selected articles were recalculated to equivalent anti-inflammatory doses of methylprednisolone, to facilitate comparison; doses of orally administered dexamethasone were decreased by $20 \%$ to adjust the dose according to the bioavailability difference between oral and intravenous administration and local injection. Where possible metaanalysis was performed using an inverse variance method, and results summarised using forest plots and relative risks, estimated based on fixed and random effects models. Heterogeneity was quantified. Results In oral surgery most clinical trials showed a significant decrease in oedema $(p=<0.0001)$ after $C S$, and local injection of methylprednisolone $\geq 25 \mathrm{mg}$ was expected to result in a significant decrease in oedema. Regarding the analgesic effect, several clinical trials showed a decrease in pain after CS $(\mathrm{P}<0.0001)$. Furthermore, CS administration resulted in a slightly higher risk of infection (relative risk 1.0041 [95\% Cl $0.9451,1.0669]$ ), but with a $p$ value of 0.89 . In orthognathic surgery methylprednisolone $\geq 85 \mathrm{mg}$ administered intravenously seemed sufficient to produce a significant decrease in oedema, and several trials pointed toward a neuroregeneration effect, but no statistical analysis could be performed. Regarding the risk of other side effects, in oral surgery a minimal risk of chronic adrenal suppression was seen: in orthognathic surgery an elevated risk of avascular osteonecrosis, steroid-induced psychosis and adrenal suppression was seen. There were no reports of decreased healing. Conclusions These findings suggest that the administration of CS in oral surgery decreases oedema and pain significantly, with no higher risk of infection and with a minimum risk of other side effects.

\section{Commentary}

The authors have made an important contribution to the current literature regarding the effectiveness and safety of perioperative corticosteroid administration in the setting of oral and maxillofacial operations. My comments regarding the paper have more to do with style rather than content:

1. Given the manuscript's audience, the introduction could be more concise.

2. When reviewing a clinical topic it is valuable to state the purpose in terms of a clinical question and then follow with specific aims. For example, 'Among patients undergoing elective oral and maxillofacial operations, do those receiving perioperative corticosteroids, when compared to those who do not, have improved outcomes in terms of oedema, pain and neuroregeneration?' The specific aims of the study were to estimate: a) therapeutic efficacy of perioperative corticosteroid administration in the setting of dentoalveolar operations and orthognathic procedures, b) minimum effective corticosteroid dose and c) risk of side-effects of perioperative corticosteroids.

3. The materials and methods were insufficiently described. The Cochrane Collaboration has detailed methods for conducting a systematic review (www.cochrane-handbook.org). Deviations from the recommendations should be addressed in the methods, and implications should be reviewed in the discussion. It takes considerable resources to conduct a 'Cochrane-style' systematic review. If the resources are not available, I recommend calling the review comprehensive, rather than systematic. The current paper fits the category of comprehensive rather than systematic. Only two sources, PubMed and the Cochrane Database, were used to identify articles. The inclusion criteria for the studies were vague. The process for including articles was not verified and there was not formal assessment of bias. Given the information provided in the methods, it would be difficult for an independent reviewer to execute and reproduce the search results and article selection.

4. The major challenge in conducting systematic or comprehensive literature reviews on topics in oral and maxillofacial surgery is the quality of the literature. Few studies qualify for inclusion in a meta-analysis. Regardless, the authors have done a good job of summarising the available data, using tables that simultaneously grouped papers based on two important predictor variables; dose and timing of administration, and using forest plots to provide summary statistics. While forest plots or other summary data are expected in meta-analyses, it is not uncommon to find the analyses missing in studies that describe themselves as 'meta-analyses'. 
5. The authors confirmed findings from previous studies about the effectiveness of corticosteroids to have a mild to moderate effect in terms of reducing postoperative oedema and pain. ${ }^{1}$ The information regarding neuroregeneration was intriguing but needs more study. The safety of perioperative corticosteroids is well established, and the study added to the body of literature on safety. The dose recommendations were helpful and confirmed my choice for dentoalveolar procedures and will change my practice for maxillofacial operations.
Thomas B. Dodson

Department of Oral and Maxillofacial Surgery, Harvard School of Dental Medicine, and Massachusetts General Hospital, Boston, Massachusetts, USA

1. Markiewicz MR, Brady MF, Ding EL, Dodson TB. Corticosteroids reduce postoperative morbidity after third molar surgery: a systematic review and meta-analysis. J Oral Maxillofac Surg 2008; 66: 1881-1894.

Evidence-Based Dentistry (2011) 12, 49-50. doi:10.1038/sj.ebd.6400794 